\title{
THE GEODESIC FLOW ON NILMANIFOLDS ASSOCIATED TO GRAPHS
}

\author{
GABRIELA P. OVANDO
}

\begin{abstract}
We study the geodesic flow on nilmanifolds associated to graphs. We are interested in the construction of first integrals to show complete integrability on some compact quotients. We start on the corresponding Lie group equipped with a left-invariant metric, which is induced to the quotients. Also examples of integrable geodesic flows and of non-integrable ones are shown.
\end{abstract}

\section{INTRODUCTION}

In this work we deal with the integrability of the geodesic flow on nilmanifolds. The condition of integrability imposes obstructions to the topology of the supporting manifold [21, 22. In known examples constructed by starting with a Lie group $N$, one is able to construct a set of linearly independent first integrals in involution on the tangent space $T N$, and doing some extra work, one can induce the first integrals to $T(\Gamma \backslash N)$, for a cocompact lattice $\Gamma<N$. These first integrals cannot be analytic in most of these examples. And in any case, finding enough functions in involution requires a case-by-case process by hand, which shows that the integrability question is not well understood. Even under restricted conditions, for instance on locally homogeneous manifolds, there is no general theory to decide the complete integrability of the geodesic flow. In [14] the authors studied some relationships between the algebra of first integrals and the algebraic geometric structure of the underlying Lie group, more specifically the corresponding isometry group.

Lie groups and their compact quotients were already used to answer nice geometrical questions [1, 2, 4, 3, 12, 18, 19. For 2-step nilpotent Lie groups, Butler introduced in [5] the notion of non-integrable Lie algebras, and proved that the geodesic flow on compact quotient manifolds arising from Lie groups with non-integrable Lie algebras cannot be integrable. It is not hard to see that non-integrable Lie algebras are singular. In the same work Butler proved non-commutative integrability for manifolds associated to almost non-singular Lie algebras. Since Bolsinov and Jovanović [1] proved that integrability in the non-commutative sense implies

2010 Mathematics Subject Classification. 70G65, 70H05, 70H06, 22E70, 22E25.

Key words and phrases. Geodesic flow; integrability; first integrals; nilmanifolds; nilpotent Lie groups.

Work partially supported by ANPCyT, CONICET, and SCyT (UNR). 
Liouville integrability, one gets the integrability of the geodesic flow on manifolds $T(\Gamma \backslash N)$, where $N$ is associated to an almost non-singular Lie algebra. Thus we have some questions between the type of the Lie algebra $\mathfrak{n}$, as non-singular, almost nonsingular, or singular, and the integrability of the geodesic flow on $M:=T(\Gamma \backslash N)$ :

$\mathfrak{n}$ almost non-singular $\Longrightarrow$ integrability on $M$;

$\mathfrak{n}$ non-integrable $\Longrightarrow$ the geodesic flow on $M$ non-integrable.

$\mathfrak{n}$ non-integrable $\Longrightarrow \mathfrak{n}$ singular.

In this work we study the integrability question on a family of nilmanifolds arising from graphs. This family was introduced by Dani and Mainkar in [8] to study Anosov automorphisms on nilmanifolds. More recently, in [9] the authors find Heisenberg-like algebras in this family and they give conditions on the graph $G$ and on a lattice $\Gamma \subset N$ for which the compact quotient $\Gamma \backslash M$ has a dense set of smoothly closed geodesics.

We start with preliminaries to study the geodesic flow on nilmanifolds. The structure of $N$ is used when indentifying $T N$ with $T^{*} N$ via the metric. More details can be found in [14. Summarizing, we get the following results:

- There is a family of compact manifolds $M=\Gamma \backslash N$ with integrable geodesic flow such that the Lie algebra of $N$ is singular. They are associated to the star graphs on $(k+1)$-vertices, $S_{k}$, for $k \geq 3$. For $k=2$ the Lie algebra arising from the graph is the Heisenberg Lie algebra of dimension three. Topologically the compact quotients are $S^{1}$-fiber bundles over $T^{2 k}$.

- Nilmanifolds $\Gamma \backslash N$ for $N$ constructed from complete graphs on $s$-vertices, $K_{s}$, are:

- Non-integrable for $s=2 n+1$; this generalizes the example in [5].

- Almost non-singular for $s=2 n$.

- Let $G$ denote a connected graph on $k$ vertices with $k \leq 4$. Then, except for the complete graph $K_{3}$, any 2-step nilpotent Lie group $N_{G}$, as well as the corresponding compact quotient, admits a completely integrable geodesic flow.

- For the graph $G=K_{3}$, we prove that the geodesic flow on the corresponding manifold $T N_{G}$ is completely integrable, although it cannot be completely integrable on $T\left(\Gamma \backslash N_{G}\right)$ for any discrete cocompact subgroup $\Gamma<N_{G}[5,7]$. We find five linearly independent invariant functions.

- For the path graph in four vertices $P$, although integrability of the geodesic flow can be derived from the almost singularity property, we explicitly show a set of first integrals in involution on $T N_{P}$.

Tools and techniques used here have no direct relationship with the previous theory developped in the 80s. We refer to Hamiltonian sytems constructed with an algebraic data as in the Adler-Kostant-Symes scheme [15, 20], or the work by Thimm concerning the geodesic flow, see 23 . In the present paper first integrals are constructed either related to Killing vectors or as invariant functions. The invariant notion is attached to the natural action of the Lie group $N$ on $T N$ induced by translations on the left by elements of the group. Notice that invariant functions are 
trivially induced to any tangent space $T(\Gamma \backslash N)$. The geometry of 2-step nilpotent Lie groups equipped with a left-invariant metric as well as the geometry around closed geodesics on compact quotients was extensively studied in [10].

In most examples given here, we show explicit computations, in the framework of Lie groups and Lie algebras. All these objects are assumed over the real numbers.

\section{Preliminaries}

In this section we recall basic notions for the study of the geodesic flow and we introduce the tools to study the geometry of 2-step nilpotent Lie groups when equipped with a left-invariant metric. In fact, this is determined at the Lie algebra level. We are interested in Lie algebras which can be constructed from graphs.

2.1. The geodesic flow on nilmanifolds. Here we consider the geodesic flow on Lie groups equipped with a left-invariant metric. We recall the general setting.

Let $T N$ denote the tangent bundle of the Lie group $N$. The geodesic field is related to the Hamiltonian vector field of the energy function $E: T N \rightarrow \mathbb{R}$ (see for instance [11]),

$$
E(p, Y)=\frac{1}{2}\langle Y, Y\rangle \text {. }
$$

We identify $T N$ with $N \times \mathfrak{n}$. In fact, for $p \in N$ and $v_{p} \in T_{p} N$ we associate the pair $(p, Y) \in N \times \mathfrak{n}$, where $Y$ is the left-invariant vector field on $N$ such that $Y(p)=v_{p}$. Therefore making use of these identifications one also has

$$
T_{(p, Y)}(T N) \simeq \mathfrak{n} \times \mathfrak{n}=\{(U, V): U, V \in \mathfrak{n}\} .
$$

The manifold $T N$ is considered with the product metric

$$
\left\langle(U, V),\left(U^{\prime}, V^{\prime}\right)\right\rangle_{(p, Y)}=\left\langle U, U^{\prime}\right\rangle+\left\langle V, V^{\prime}\right\rangle,
$$

and it has a canonical symplectic structure which is induced by the canonical symplectic structure on $T^{*} N$ via the metric. This gives the definition of a Poisson bracket on $C^{\infty}(T N)$, denoted by $\{$,$\} , as$

$$
\{f, g\}=\Omega\left(X_{f}, X_{g}\right), \quad \text { for } f, g \in C^{\infty} T N,
$$

where $\Omega$ is the canonical symplectic form on $T N$, and $X_{f}, X_{g}$ are the Hamiltonian vector fields of $f$ and $g$, respectively. Recall that for a smooth function $h: T N \rightarrow \mathbb{R}$, the Hamiltonian vector field of $h$, denoted by $X_{h}$, is implicitly given by

$$
d h_{(p, Y)}(U, V)=\Omega_{(p, Y)}\left(X_{h}(p, Y),(U, V)\right) .
$$

On the other hand, the gradient field of $h$, denoted by $\operatorname{grad} h$, is given by the formula

$$
d h_{(p, Y)}(U, V)=\left\langle\operatorname{grad}_{(p, Y)} h,(U, V)\right\rangle .
$$

The geodesic field on $T N$ is the vector field associated with the geodesic flow

$$
\Phi_{t}(p, Y)=\gamma^{\prime}(t),
$$

where $\gamma(t)$ is the geodesic on $N$ with initial conditions $\gamma(0)=p, \gamma^{\prime}(0)=(p, Y)$. 
We say that a smooth function $f: T N \rightarrow \mathbb{R}$ is a first integral of the geodesic flow if the derivative of $f$ in the direction of $X_{E}$ vanishes: $X_{E}(f)=0$; equivalently, if

$$
\{f, E\}=0 \text {. }
$$

Notice that the gradient field of the energy function for a left-invariant metric is $\operatorname{grad} E(p, Y)=(0, Y)$. The proof of the following proposition arises from the definitions above; see [14].

Proposition 2.1. Let $N$ denote a Lie group equipped with a left-invariant metric. Let $f, g \in C^{\infty}(T N)$ be smooth functions with $\operatorname{grad}_{(p, Y)} f=(U, V)$ and $\operatorname{grad}_{(p, Y)} g=$ $\left(U^{\prime}, V^{\prime}\right)$. Then

(i) the Hamiltonian vector field for a smooth $f: T N \rightarrow \mathbb{R}$ is

$$
X_{f}(p, Y)=\left(V \operatorname{ad}^{t}(V)(Y)-U\right),
$$

where $\operatorname{ad}^{t}(V)$ denotes the transpose of $\operatorname{ad}(V)$ with respect to the metric on $\mathfrak{n}$; (ii) the Poisson bracket follows

$$
\{f, g\}(p, Y)=\left\langle U, V^{\prime}\right\rangle-\left\langle V, U^{\prime}\right\rangle+\left\langle Y,\left[V^{\prime}, V\right]\right\rangle .
$$

Let $f, g: T N \rightarrow \mathbb{R}$ be smooth functions. We say that they are in involution whenever they Poisson commute:

$$
\{f, g\}=0 \text {. }
$$

The definition above says that $\{f, g\}=0$ if and only if $d f\left(X_{g}\right)=0$ if and only if $X_{g}(f)=0$ (and also $X_{f}(g)=0$ ). Therefore $f$ is constant along integral curves of $X_{g}$ (analogously for $X_{f}$ ).

The question of explicitly finding functions in involution is a research topic with several open questions. If $M$ is a Riemannian manifold and $X^{*}$ is a Killing vector field on $M$, then the function $f_{X^{*}}: T M \rightarrow \mathbb{R}$ defined as $f_{X^{*}}(v)=\left\langle X^{*}(\pi(v)), v\right\rangle$ is a first integral of the geodesic flow. But in general it is not clear if one can produce enough functions in involution for proving the complete integrability.

We shall discuss later the question of invariant functions. Indeed, a Lie group $N$ acts on its tangent bundle $q \cdot v_{p}=d L_{q} v_{p}$, that in terms of the identification above gives

$$
q \cdot(p, Y)=(q p, Y)
$$

A function $f: T N \rightarrow \mathbb{R}$ is called invariant if $f(q p, Y)=f(p, Y)$ for all $p, q \in N$, that is, $f$ is invariant under the action of $N$.

Definition 2.2. We say that a Riemannian manifold $(M,\langle\rangle$,$) has completely in-$ tegrable geodesic flow (in the sense of Liouville) if there exist $n$ first integrals of the geodesic flow, $f_{i}: T M \rightarrow \mathbb{R}$, where $n=\operatorname{dim} M$, such that $\left\{f_{i}, f_{j}\right\}=0$ for all $i, j$ and the gradients of $f_{1}, \ldots, f_{n}$ are linearly independent on an open dense subset of $T M$.

Integrability or non-integrability are difficult questions. Integrability imposes topological restrictions on compact manifolds. 
Let $N$ denote a Lie group equipped with a left invariant metric $g$. We say that $\Lambda \subset N$ is a lattice if $\Lambda$ is a discrete subgroup such that the quotient $\Lambda \backslash N$ is a compact space. Sometimes one also says that $\Lambda$ is a discrete cocompact subgroup of $N$.

Mal'cev [17] has shown that a simply connected nilpotent Lie group $N$ admits a lattice if and only if its Lie algebra admits a basis with rational structure constants. In this case the metric $g$ is induced to the quotient and is also denoted by $g$. It satisfies

$$
g\left(d p_{m} X, d p_{m} Y\right)=g(X, Y), \quad \text { for } X, Y \in \mathfrak{n}, m \in N,
$$

where $p: N \rightarrow \Lambda \backslash N$ is the canonical projection. One looks for first integrals not only on $N$ but on the compact quotients $\Lambda \backslash N$.

Theorem 2.3 ([7, Theorem 1.3]). Let $\mathfrak{n}$ be a non-integrable 2-step nilpotent Lie algebra with associated simply connected Lie group $N$. Assume that there exists a discrete, cocompact subgroup $\Lambda$ of $N$. Then for any such $\Lambda$ and any left-invariant metric $g$ on $N$, the geodesic flow of $(\Lambda \backslash N, g)$ is not completely integrable.

The precise definition of non-integrable 2-step nilpotent Lie algebra will be given in the next subsection.

2.2. 2-step nilpotent Lie groups and graphs. Let $N$ denote a 2-step nilpotent Lie group equipped with a left-invariant metric $\langle$,$\rangle . Its Lie algebra \mathfrak{n}$ decomposes as the orthogonal direct sum

$$
\mathfrak{n}=\mathfrak{v} \oplus \mathfrak{z},
$$

where $\mathfrak{v}=\mathfrak{z}^{\perp}$ and $\mathfrak{z}$ is the center of $\mathfrak{n}$. In this situation each element $Z \in \mathfrak{z}$ induces a skew-symmetric linear map on $\mathfrak{v}, j(Z): \mathfrak{v} \rightarrow \mathfrak{v}$, given by

$$
\langle j(Z) U, V\rangle=\langle[U, V], Z\rangle
$$

for all $U, V \in \mathfrak{v}$. The geometry of $N$ is encoded in the maps $j(Z)[10$.

Recall that whenever $N$ is simply connected, the exponential map, exp $: \mathfrak{n} \rightarrow N$, is a diffeomorphism with inverse map $\log : N \rightarrow \mathfrak{n}$. Moreover, one has the formula

$$
\exp (X) \exp (Y)=\exp \left(X+Y+\frac{1}{2}[X, Y]\right), \quad \text { for all } X, Y \in \mathfrak{n} .
$$

This formula enables the realization of the Lie group at the Lie algebra level. In fact, via the exponential map we define a product on the Lie algebra: take the left-invariant vector fields $X, Y \in \mathfrak{n}$ and define a product $X \cdot Y$ by $X+Y+\frac{1}{2}[X, Y]$. In this way the exponential map, as the only one-parameter group on $N$ with initial condition $X \in \mathfrak{n}$, becomes the map that, whenever $X=\sum_{i} x_{i} X_{i}$ for a basis of vector fields $\left\{X_{i}\right\}$, sends $X$ to the vector $\left(x_{1}, x_{2}, \ldots, x_{n}\right)$ in $\mathbb{R}^{n}$, in usual coordinates.

In particular, for a 2-step nilpotent Lie group, after equations $(2.1)$ and 2.2 , one has:

- For the energy function $E: T N \rightarrow \mathbb{R}$, its Hamiltonian vector field is

$$
X_{E}(p, Y)=\left(Y, j\left(Y_{\mathfrak{z}}\right) Y_{\mathfrak{v}}\right) .
$$


- A function $f: T N \rightarrow \mathbb{R}$ with gradient $\operatorname{grad} f(p, Y)=(U, V)$ is a first integral of the geodesic flow on $T N$ if and only if

$$
\langle Y, U\rangle=\left\langle j\left(Y_{\mathfrak{z}}\right) V_{\mathfrak{v}}, Y_{\mathfrak{v}}\right\rangle .
$$

A distinguished family of 2-step nilpotent Lie algebras can be constructed starting with a graph $G$. Let $G$ be a directed graph with at least one edge. Denote the vertices of $G$ by $S=\left\{X_{1}, \ldots, X_{m}\right\}$ and its edges by $E=\left\{Z_{1}, \ldots, Z_{q}\right\}$.

The Lie algebra $\mathfrak{n}_{G}$ is the vector space direct sum $\mathfrak{n}_{G}=\mathfrak{v} \oplus \mathfrak{z}$, where we let $E$ be a basis over $\mathbb{R}$ for $\mathfrak{z}$ and $S$ be a basis over $\mathbb{R}$ for $\mathfrak{v}$. Define the bracket relations among elements of $S$ according to adjacency rules:

- if $Z_{k}$ is a directed edge from vertex $X_{i}$ to vertex $X_{l}$ then define the skewsymmetric bracket $\left[X_{i}, X_{l}\right]=Z_{k}$.

- If there is no edge between two vertices, then define the bracket of those two elements in $S$ to be zero.

Extend the bracket relation to all of $\mathfrak{v}$ by using bilinearity of the bracket.

Remark 2.4. In [16] it was proved that the 2-step nilpotent Lie algebras associated with two directed graphs are Lie isomorphic if and only if the graphs from which they arise are isomorphic.

Choose the inner product on $\mathfrak{n}_{G}$ so that $S \cup E$ is an orthonormal basis for $\mathfrak{n}_{G}$. Observe that if $Z_{k}$ is a directed edge from $X_{i}$ to $X_{l}$ then the map $j\left(Z_{k}\right)$ defined in equation (2.3) satisfies $j\left(Z_{k}\right) X_{i}=X_{l}$ and $j\left(Z_{k}\right) X_{p}=0$ for any other $X_{p} \in S$ where $p \neq i, l$.

Example 2.5. The star graph $S_{k}$ has $k+1$ vertices $V_{0}, V_{1}, \ldots, V_{k}$ and edges $Z_{i}$. The vertices $V_{0}$ and $V_{i}$ are joined by the edge $Z_{i}$, so that the Lie bracket gives $\left[V_{0}, V_{i}\right]=Z_{i}$. Setting $Z=a_{1} Z_{1}+\cdots+a_{k} Z_{k}$, the matrix presentation of $j(Z): \mathfrak{v} \rightarrow \mathfrak{v}$ in the basis $V_{0}, V_{1}, \ldots, V_{k}$ is given by

$$
\left(\begin{array}{ccccc}
0 & -a_{1} & -a_{2} & \ldots & -a_{k} \\
a_{1} & 0 & 0 & \ldots & 0 \\
a_{2} & 0 & 0 & \ldots & 0 \\
\vdots & \vdots & \vdots & \ddots & \vdots \\
a_{k} & 0 & 0 & \ldots & 0
\end{array}\right) .
$$

Therefore $j(Z)$ is singular for $k+1>2$, that is for the star graph with $k+1>2$ vertices.

Example 2.6. The complete graph on $n$ vertices $K_{n}$ is the graph that has an edge between every pair of distinct vertices. The graph $K_{2}$ corresponds to the known Heisenberg Lie algebra of dimension three; it has two vertices $V_{1}, V_{2}$ and one edge $Z$,

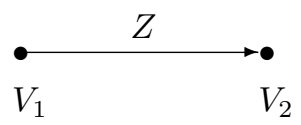


so that the corresponding map $j(Z)$ is non-singular. The complete graph $K_{3}$ has three vertices $V_{1}, V_{2}, V_{3}$ and three edges $Z_{1}, Z_{2}, Z_{3}$.

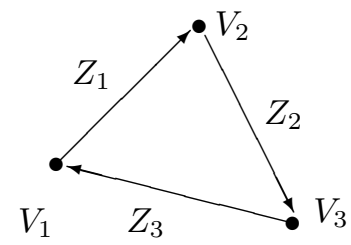

Let $\mathfrak{n}_{K_{3}}$ denote the corresponding 2-step nilpotent Lie algebra where we have the Lie brackets

$$
\left[V_{1}, V_{2}\right]=Z_{1}, \quad\left[V_{2}, V_{3}\right]=Z_{2}, \quad\left[V_{3}, V_{1}\right]=Z_{3} .
$$

Let $\langle$,$\rangle denote the metric on \mathfrak{n}_{K_{3}}$ for which this basis is orthonormal. The map $j(Z): \mathfrak{v} \rightarrow \mathfrak{v}$ has a matrix presentation in the basis $\left\{V_{1}, V_{2}, V_{3}\right\}$ of $\mathfrak{v}$ given by

$$
j\left(a Z_{1}+b Z_{2}+c Z_{3}\right)=\left(\begin{array}{ccc}
0 & -a & -c \\
a & 0 & -b \\
c & b & 0
\end{array}\right) .
$$

Notice that the dimension $\operatorname{ker} j(Z)=1$, for every $Z \in \mathfrak{z}-\{0\}$. More generally, consider the complete graph on $n$ vertices, $K_{n}$. The dimension of the center is $\operatorname{dim} \mathfrak{z}=\left(\begin{array}{l}n \\ 2\end{array}\right)=\operatorname{dim} \mathfrak{s o}(n)$. Thus,

- if $n$ is odd, every $j(Z)$ is a singular map for every $Z \in \mathfrak{z}$;

- if $n$ is even, there exists $Z \in \mathfrak{z}$ such that $j(Z)$ is non-singular. In fact, assuming $V_{1}, V_{2}, \ldots, V_{2 s}$ are vertices and $Z_{i j}=\left[V_{i}, V_{j}\right]$, take the non-singular map $j(Z)$ with matrix

$$
j\left(Z_{12}+Z_{34}+\cdots+Z_{2 s-1,2 s}\right)=\left(\begin{array}{cccccccc}
0 & -1 & & & & & \\
1 & 0 & & & & & \\
& & 0 & -1 & & & \\
& & 1 & 0 & & & \\
& & & & \ddots & & \\
& & & & & 0 & -1 \\
& & & & & 1 & 0
\end{array}\right) .
$$

We say that a 2 -step nilpotent Lie algebra $\mathfrak{n}$ is

- non-singular if $\operatorname{ad}(X): \mathfrak{n} \rightarrow \mathfrak{z}$ is surjective for all $X \notin \mathfrak{z}$;

- almost non-singular if $j(Z)$ is non-singular for every $Z$ in an open dense subset of $\mathfrak{z}$

- singular if $j(Z)$ is singular for all $Z$ in $\mathfrak{z}$.

Every 2-step nilpotent Lie algebra belongs to one and only one of the types non-singular, almost non-singular, or singular [13]. 


\section{Notice that}

- the fact of $\mathfrak{n}$ being non-singular is equivalent to asking $j(Z)$ to be nonsingular for any $Z \in \mathfrak{z}-\{0\}$ for a (any) metric on $\mathfrak{n}$ (in fact, this does not depend on the choice of the left-invariant metric; see for instance [10]);

- whenever a 2-step nilpotent Lie algebra $\mathfrak{n}$ is equipped with a metric, if there are two nonzero elements $Z, Z^{\prime} \in \mathfrak{z}$ such that $j(Z)$ is non-singular and $j\left(Z^{\prime}\right)$ is singular, then the Lie algebra $\mathfrak{n}$ is almost non-singular.

Remark 2.7. Assume that $G$ is a graph with at least one edge. If the graph $G$ is isomorphic to the complete graph $K_{2}$ then its Lie algebra $\mathfrak{n}_{G}$ is non-singular (this Lie algebra is also isomorphic to the Heisenberg Lie algebra of dimension three).

Assume that $G$ is not isomorphic to $K_{2}$. Thus $G$ contains an edge $Z$ and a vertex $X$ such that the edge $Z$ is not incident to it. Then $j(Z) X=0$, and therefore $\mathfrak{n}_{G}$ is either almost non-singular or singular. Lemma 3.3 in [9] proved that the Lie algebra $\mathfrak{n}_{G}$ is non-singular if and only if $G=K_{2}$.

Further if $G$ has more than one connected component, $\mathfrak{n}_{G}$ is almost non-singular if and only if each connected component is either non-singular or almost nonsingular [9].

Definition 2.8. Let $\mathfrak{n}$ be a 2-step nilpotent Lie algebra, $\mathfrak{n}^{*}$ its dual space, and for $\lambda \in \mathfrak{n}^{*}$ let $\operatorname{ad}^{*}(X) \lambda$ denote the element in $\mathfrak{n}^{*}$ given by $\operatorname{ad}^{*}(X) \lambda(Y)=-\lambda([X, Y])$, for all $X, Y \in \mathfrak{n}$.

(i) For $\lambda \in \mathfrak{n}^{*}$, let $\mathfrak{n}_{\lambda}:=\left\{X \in \mathfrak{n}: \operatorname{ad}^{*}(X) \lambda=0\right\}$.

(ii) A $\lambda \in \mathfrak{n}^{*}$ is called regular if $\mathfrak{n}_{\lambda}$ has minimal dimension.

(iii) A pair $\mu, \lambda \in \mathfrak{n}^{*}$ is called generic if $\operatorname{dim}\left[\mathfrak{n}_{\lambda}, \mathfrak{n}_{\mu}\right]$ is minimal. The Lie algebra $\mathfrak{n}$ is called non-integrable if for a dense open subset of generic pairs $\lambda, \mu$, one has $\left[\mathfrak{n}_{\lambda}, \mathfrak{n}_{\mu}\right] \neq 0$.

Remark 2.9. Let $\lambda \in \mathfrak{n}^{*}$. Indeed, $\mathfrak{n}_{\lambda}$ is the isotropy algebra for the coadjoint representation: $p \cdot \lambda=-\lambda \circ \operatorname{Ad}\left(g^{-1}\right)$, for $p \in N$. Notice that $\lambda$ is regular whenever the dimension of the orbit of $\lambda$ under the coadjoint representation is maximal. It is clear that the center $\mathfrak{z} \subset \mathfrak{n}$ is contained in $\mathfrak{n}_{\lambda}$ for any $\lambda \in \mathfrak{n}^{*}$.

We can read the non-integrability notion making use of tools at the Lie algebra level. Let $\langle$,$\rangle denote an inner product on the Lie algebra \mathfrak{n}$, with respect to which there is the following splitting as direct sum of vector spaces $\mathfrak{n}=\mathfrak{v} \oplus \mathfrak{z}$, with $\mathfrak{v}=\mathfrak{z}^{\perp}$.

For any $\lambda \in \mathfrak{n}^{*}$ there exists unique $Z \in \mathfrak{z}$ and $V \in \mathfrak{v}$ such that $\lambda=\ell_{V+Z}$, where $\ell_{V+Z}(X)=\langle V+Z, X\rangle$. Thus we denote $\mathfrak{n}_{\ell_{V+Z}}$ directly by $\mathfrak{n}_{V+Z}$. So

$$
\begin{aligned}
\mathfrak{n}_{V+Z} & =\{X \in \mathfrak{n}:\langle V+Z, \operatorname{ad}(X) U\rangle=0 \text { for all } U \in \mathfrak{n}\} \\
& = \begin{cases}\mathfrak{n} & \text { if } Z=0, \\
\mathfrak{z} \oplus \operatorname{ker} j(Z) & \text { if } Z \neq 0 .\end{cases}
\end{aligned}
$$

Assume that $\ell_{V+Z}$ is regular; thus for $\mathfrak{n}$ non-singular or almost non-singular, it is clear that $\mathfrak{n}_{V+Z}=\mathfrak{z}$. In both cases, for both $\lambda, \mu$ regular, one has $\left[\mathfrak{n}_{\lambda}, \mathfrak{n}_{\mu}\right]=0$.

Corollary 2.10. A 2-step nilpotent non-integrable Lie algebra is singular. 
Example 2.11. In [5] Butler showed an example of a non-integrable Lie algebra. Its Lie group has Lie algebra isomorphic to $K_{3}$.

More generally, assume that $n$ is odd, $n=2 k+1>2$, and $G=K_{n}$ is the complete graph. As mentioned above the Lie algebra $\mathfrak{n}_{G}$ is singular. Roughly speaking, the graph $K_{2 k+1}$ can be constructed from the complete graph $K_{2 k}$ by adding one vertex and all the edges joining the added vertex with the previous ones.

Let $S$ denote the set of vertices, assume $|S|=n$ with $n=2 k+1$, and choose the vertex $V_{2 k+1} \in S$. Denote by $Z_{i j}$ (or $Z_{i, j}$ ) the basis element in $\mathfrak{z}$ such that $Z_{i, j}:=\left[V_{i}, V_{j}\right]$.

Take $Z \in \mathfrak{z}$ defined as $Z:=\sum_{i=1}^{k} Z_{2 i-1,2 i} \in \mathfrak{z}$. Then the restriction of $j(Z)$ to the vector subspace $\mathfrak{w}_{1}$ spanned by $V_{1}, V_{2}, \ldots, V_{2 k}$ - see matrix in Example 2.6 -is a non-singular linear map, and $V_{2 k+1} \in \operatorname{ker} j(Z)$ since $j\left(Z_{2 i-1,2 i}\right) V_{2 k+1}=0$ for all $i$.

Analogously, take $V_{1}$ in the kernel of $j(\tilde{Z})$ for $\tilde{Z}=\sum_{i=1}^{k} Z_{2 i, 2 i+1}$ so that the restriction of $j(\tilde{Z})$ is non-singular on the vector subspace $\mathfrak{w}_{2}$ of dimension $2 k$ spanned by $V_{2}, V_{3}, \ldots, V_{2 k+1}$. It is clear that $\ell_{Z}$ and $\ell_{\tilde{Z}}$ are regular in $\mathfrak{n}_{G}^{*}$. Moreover, $V_{1} \in \mathfrak{n}_{\tilde{Z}}$, $V_{2 k+1} \in \mathfrak{n}_{Z}$, and $\left[V_{1}, V_{2 k+1}\right]=Z_{1,2 k+1}$. This implies that $K_{2 k+1}$ is non-integrable.

Proposition 2.12. Let $n=2 k+1 \in \mathbb{N}$ with $k \geq 1$, and let $G=K_{n}$ denote the complete graph on $n$ vertices. The corresponding Lie algebra $\mathfrak{n}_{G}$ is non-integrable.

The proposition above and Theorem 1.3 in [7] imply that the 2-step nilpotent Lie group $N_{G}$, constructed by starting with the complete graph $G=K_{n}$, with $n$ odd, has no completely integrable geodesic flow on $\Lambda \backslash N_{G}$, for any left-invariant metric and any lattice $\Lambda \subset N_{G}$.

Example 2.13. Connected graphs with $n$ vertices and the type of their Lie algebras:

(i) $n=2$ : the complete $K_{2}, \mathfrak{n}_{K_{2}}$ non-singular;

(ii) $n=3$ : the complete $K_{3}$, with $\mathfrak{n}_{K_{3}}$ singular, the star graph $S_{3}$ with $\mathfrak{n}_{S_{3}}$ singular;

(iii) $n=4$ : the star graph $S_{4}$ with $\mathfrak{n}_{S_{4}}$ singular and the (corresponding to) almostnon-singular Lie algebras: the complete graph $K_{4}$, the cycle $C_{4}$, the path in four vertices $P_{4}$, and the graphs $G_{1}$ and $G_{2}$. See $[9$.
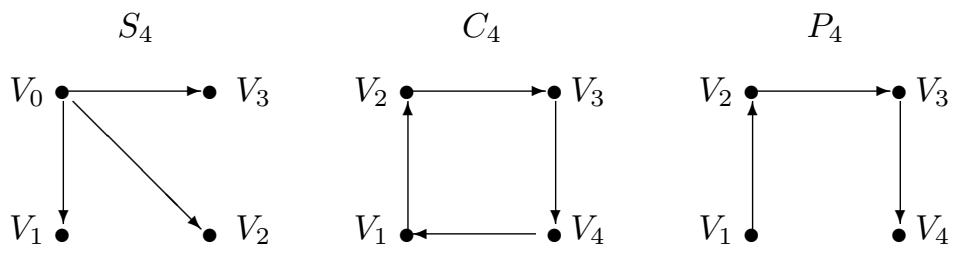

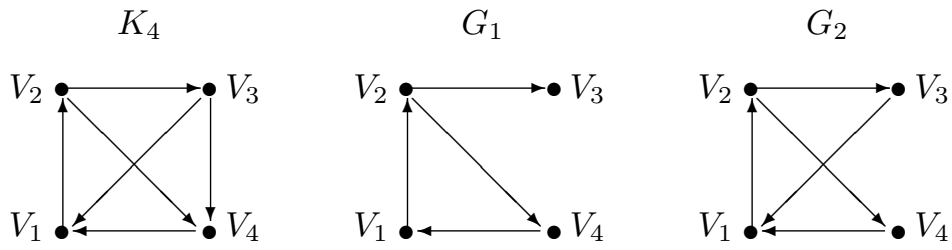

\section{INVOLUTION OF INVARIANT FUNCTIONS}

The goal now is the study of invariant functions. Invariant functions descend to any compact quotient $\Lambda \backslash N$ for any lattice $\Lambda \subset N$. Therefore it is desirable to have a good number of independent invariant functions.

Under the natural action of $N$ on $T N \simeq N \times \mathfrak{n}$ given by $n \cdot(p, Y)=(n p, Y)$, a function $f: T N \rightarrow \mathbb{R}$ is invariant if $f(p, Y)=f(e, Y)$ for all $p \in N, Y \in \mathfrak{n}$. For instance if the metric on the Lie group $N$ is left-invariant, the corresponding energy function is invariant.

Concerning invariant functions in $C^{\infty}(T N)$ for a Lie group $N$, from notions and properties above one proves the following statements.

(i) The gradient field for an invariant function $f: T N \rightarrow \mathbb{R}$ has the form

$$
\operatorname{grad}_{(p, Y)}(f)=(0, V)
$$

for some $V \in \mathfrak{n}$. In fact, denote by $U, V$ the components of the gradient: $\operatorname{grad} f(p, Y)=(U, V)$. Since $f(e, Y)=f(p, Y)$, one has $d f_{(p, Y)}\left(U^{\prime}, V^{\prime}\right)=$ $\left.\frac{d}{d s}\right|_{s=0} f(e, Y+s V)=\left\langle U^{\prime} U^{\prime}\right\rangle+\left\langle V, V^{\prime}\right\rangle$, so that $U=0$. The corresponding Hamiltonian vector field is given by

$$
X_{f}(p, Y)=\left(V, \operatorname{ad}^{t}(V) Y\right)
$$

where $\operatorname{ad}^{t}(V)$ denotes the transpose of $\operatorname{ad}(V)$ relative to the metric on $\mathfrak{n}$. In particular, an invariant function $f: T N \rightarrow \mathbb{R}$ is a first integral of the geodesic flow if and only if

$$
0=\langle Y,[V, Y]\rangle \quad \text { for }(0, V)=\operatorname{grad} f(p, Y) .
$$

(ii) The set of invariant functions $\{f: T N \rightarrow \mathbb{R}: f$ is invariant $\}$ is in correspondence with the set of functions on $\mathfrak{n}:\{F: \mathfrak{n} \rightarrow \mathbb{R}\}$.

Given an invariant function $f: T N \rightarrow \mathbb{R}$ define $F: \mathfrak{n} \rightarrow \mathbb{R}$ as $F(Y)=$ $f(e, Y)$, and conversely, given $F: \mathfrak{n} \rightarrow \mathbb{R}$ define an invariant function $f:$ $T N \rightarrow \mathbb{R}$ by

$$
f(p, Y)=F(Y) \text { for all } p \in N, Y \in \mathfrak{n} .
$$

(iii) Let $f_{1}, f_{2}: T N \rightarrow \mathbb{R}$ be invariant functions. Then their corresponding gradients follow $\operatorname{grad}\left(f_{i}\right)(p, Y)=\left(0, V_{F_{i}}\right)$ for $i=1,2$ and the Poisson bracket is

$$
\left\{f_{1}, f_{2}\right\}(p, Y)=-\left\langle Y,\left[V_{F_{1}}, V_{F_{2}}\right]\right\rangle,
$$

where $V_{F_{i}}=\operatorname{grad}_{\mathfrak{n}} F_{i}$. In fact, on the one hand $\operatorname{grad} f_{i}(p, Y)=\left(0, V_{i}\right)$, so that

$$
\left\{f_{1}, f_{2}\right\}(p, Y)=-\left\langle Y,\left[V_{1}, V_{2}\right]\right\rangle
$$


but one also knows that $\left.\frac{d}{d s}\right|_{s=0} f_{i}\left(e, Y+s V^{\prime}\right)=\left.\frac{d}{d s}\right|_{s=0} F_{i}\left(Y+s V^{\prime}\right)=$ $d F_{i_{Y}}\left(V^{\prime}\right)=\left\langle\operatorname{grad}_{\mathfrak{n}} F_{i}(Y), V^{\prime}\right\rangle$. These equations prove the equality 3.1.

The next proposition specifies some invariant functions which are first integrals of the geodesic flow. Notice that for every $(p, Y) \in T N$, one has $\operatorname{grad} f_{Z_{0}}(p, Y)=$ $\left(0, Z_{0}\right)$ and $\operatorname{grad} g_{A}(p, Y)=(0, A Y)$. See the proof in [14].

Proposition 3.1. Let $(N,\langle\cdot, \cdot\rangle)$ be a Lie group with a left-invariant metric.

(i) The function $f_{Z_{0}}: T N \rightarrow \mathbb{R}$, defined by $f_{Z_{0}}(p, Y)=\left\langle Y, Z_{0}\right\rangle$, is a first integral of the geodesic flow for all $Z_{0} \in \mathfrak{z}$. Moreover, the family $\left\{f_{Z_{0}}\right\}_{Z_{0} \in \mathfrak{z}}$ is a Poisson-commutative family of first integrals.

(ii) Let $A: \mathfrak{n} \rightarrow \mathfrak{n}$ be a symmetric endomorphism of $\mathfrak{n}$ and let $g_{A}: T N \rightarrow \mathbb{R}$ denote the quadratic polynomial given by $g_{A}(p, Y)=\frac{1}{2}\langle Y, A Y\rangle$. Then $g_{A}$ is a first integral of the geodesic flow if and only if $\langle Y,[A Y, Y]\rangle=0$ for all $Y \in \mathfrak{n}$.

Let $N$ denote a Lie group with Lie algebra $\mathfrak{n}$. The Gauss map $G: T N \rightarrow \mathfrak{n}$ is given by $G(p, Y)=Y$. Thus its differential $d G_{(p, Y)}(U, V)=V$. Geometrically the Gauss map sends an element $Y_{p} \in T N$ to $d L_{p^{-1}} Y_{p}$ and takes this as the initial value corresponding to the left-invariant vector field $Y$. A smooth function $F: \mathfrak{n} \rightarrow \mathbb{R}$ corresponds to a smooth function $f: T N \rightarrow \mathbb{R}$ defined as $f=F \circ G$. Thus for $F_{1}, F_{2} \in C^{\infty}(\mathfrak{n})$,

$$
\begin{aligned}
\left\{F_{1} \circ G, F_{2} \circ G\right\}(p, Y) & =\left\{F_{1}, F_{2}\right\} \circ G(p, Y) \\
& =-\left\langle Y,\left[V_{F_{1}}, V_{F_{2}}\right]\right\rangle .
\end{aligned}
$$

The Gauss map in this context was previously studied in [11. We notice that the Poisson bracket on the Lie algebra $\mathfrak{n}$ has symplectic leaves given by the "coadjoint" orbits, which are induced from $\mathfrak{n}^{*}$ to $\mathfrak{n}$ via the corresponding metric. However, the Gauss map $G$ does not send $(p, Y) \in T N$ to a vector in $\mathfrak{n}$ which is tangent to a coadjoint orbit, that is, one should project it if necessary.

For invariant functions, the Poisson bracket on $\mathfrak{n}$ (see [10]) is in correspondence with the Poisson bracket on $T N,\left\{f_{1}, f_{2}\right\}(p, Y)=\left\{F_{1}, F_{2}\right\}(e, Y)$, and via the relation $f_{i}(p, Y)=F_{i}(Y)=F_{i} \circ G(p, Y)$, one has $\left\{f_{1}, f_{2}\right\}(p, Y)=\left\{F_{1}, F_{2}\right\} \circ G(p, Y)$. We summarize these results below.

Proposition 3.2. Let $N$ denote a Lie group with Lie algebra $\mathfrak{n}$. Let $G: T N \rightarrow \mathfrak{n}$ denote the Gauss map.

(i) For functions $F_{1}, F_{2}: \mathfrak{n} \rightarrow \mathbb{R}$ one has $F_{i} \circ G: T N \rightarrow \mathbb{R}$, so that for every $(p, Y) \in T N$ :

$$
\left\{F_{1} \circ G, F_{2} \circ G\right\}(p, Y)=\left\{F_{1}, F_{2}\right\} \circ G(p, Y)=-\left\langle Y,\left[V_{F_{1}}, V_{F_{2}}\right]\right\rangle,
$$

where $V_{F_{i}}=\operatorname{grad}_{\mathfrak{n}} F_{i}$, so that $\operatorname{grad}\left(F_{i} \circ G\right)=\left(0, V_{F_{i}}\right)$. Clearly $F_{i} \circ G: T N \rightarrow \mathbb{R}$ is invariant by construction.

(ii) Given a smooth function $f: T N \rightarrow \mathbb{R}$ there exists $F: \mathfrak{n} \rightarrow \mathbb{R}$ so that $F \circ G=f$ if and only if $f$ is invariant. In this case we have the commutative diagram 


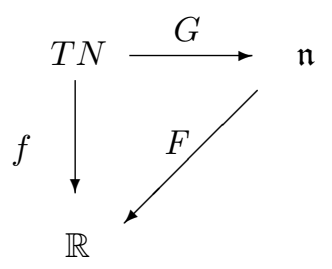

So for smooth invariant functions $f_{i}: T N \rightarrow \mathbb{R}$, with associated functions $F_{i}: \mathfrak{n} \rightarrow \mathbb{R}$, where $f_{i}=F_{i} \circ G$, for $i=1,2$, the Poisson bracket follows as in equation 3.2$):\left\{f_{1}, f_{2}\right\}(p, Y)=\left\{F_{1}, F_{2}\right\} \circ G(p, Y)$.

In the following paragraphs we shall show a construction of invariant functions for 2-step nilpotent Lie groups.

Assume that $N$ is a 2-step nilpotent Lie group with Lie algebra $\mathfrak{n}$. Let $\langle$,$\rangle denote$ a left-invariant metric on $N$ which gives the orthogonal decomposition

$$
\mathfrak{n}=\mathfrak{v} \oplus \mathfrak{z}, \quad \text { for } \mathfrak{v}=\mathfrak{z}^{\perp} .
$$

Assume that $A: \mathfrak{n} \rightarrow \mathfrak{n}$ is a symmetric linear map, preserving this decomposition. In particular, $A \mathfrak{v} \subseteq \mathfrak{v}$. Thus the map $g_{A}$ given by $g_{A}(p, Y)=\langle A Y, Y\rangle$ is a first integral of the geodesic flow if and only if

$$
\left\langle j\left(Y_{\mathfrak{z}}\right) A Y_{\mathfrak{v}}, Y_{\mathfrak{v}}\right\rangle=0, \quad \text { for all } Y=Y_{\mathfrak{z}}+Y_{\mathfrak{v}} \in \mathfrak{n},
$$

which is equivalent to

$$
j(Z) A=A j(Z), \quad \text { for all } Z \in \mathfrak{z} .
$$

Moreover, the maps $g_{A}, g_{B}$ are involution (for respective symmetric maps $A, B$ : $\mathfrak{v} \rightarrow \mathfrak{v})$ if and only if

$$
j(Z) A B=j(Z) B A, \quad \text { for all } Z \in \mathfrak{z} .
$$

See 14 for details.

L. Butler in [5] exhibited the following polynomial first integrals for the geodesic flow. The statement given in [11] is more adequate for our work here.

Proposition 3.3. Let $N$ denote an almost non-singular 2-step nilpotent Lie group equipped with a left-invariant metric $\langle$,$\rangle . Let \mathfrak{n}$ denote its Lie algebra with orthogonal splitting $\mathfrak{n}=\mathfrak{v} \oplus \mathfrak{z}$, where $\operatorname{dim} \mathfrak{v}=2 n$. For $i=1, \ldots, n$, the invariant functions $f_{i}: T N \rightarrow \mathbb{R}$ given by

$$
f_{i}(p, Y)=\left\langle V, j(Z)^{2 i} V\right\rangle, \quad Y=V+Z \in \mathfrak{n}=\mathfrak{v} \oplus \mathfrak{z},
$$

are first integrals of the geodesic flow.

Example 3.4. Let $P$ denote the graph which is the path of length three on four distinct vertices. Assume that the vertices are $V_{1}, V_{2}, V_{3}, V_{4}$ and let $\mathfrak{n}_{P}$ be the 2-step nilpotent Lie algebra associated with this graph with the Lie brackets

$$
\left[V_{1}, V_{2}\right]=Z_{1}, \quad\left[V_{2}, V_{3}\right]=Z_{2}, \quad\left[V_{3}, V_{4}\right]=Z_{3} .
$$


Assume a metric that makes of the set $V_{1}, V_{2}, V_{3}, V_{4}, Z_{1}, Z_{2}, Z_{3}$ an orthonormal basis. Then $j\left(Z_{i}\right)$ is singular for every $i$ but $j\left(Z_{1}+Z_{3}\right)$ is non-singular. Thus $\mathfrak{n}_{P}$ is almost non-singular. Note that a general $j\left(a Z_{1}+b Z_{2}+c Z_{3}\right)$ in the basis $V_{1}, V_{2}, V_{3}, V_{4}$ has a matrix of the form

$$
j(Z)=\left(\begin{array}{cccc}
0 & -a & 0 & 0 \\
a & 0 & -b & 0 \\
0 & b & 0 & -c \\
0 & 0 & c & 0
\end{array}\right) .
$$

It is not hard to prove that a symmetric map on $\mathfrak{v}$ inducing an invariant function on $T N_{P}$ of the form $g_{A}(p, Y)=\frac{1}{2}\langle A Y, Y\rangle$ gives a first integral of the geodesic flow only for $A=\mathrm{Id}$. In fact, one verifies that Id is the only solution for symmetric maps $S: \mathfrak{v} \rightarrow \mathfrak{v}$ satisfying the condition $[S, J(Z)]=0$ for all $Z \in \mathfrak{z}$. Thus we consider the function $\bar{h}: \mathfrak{n}_{P} \rightarrow \mathbb{R}$ given by $\bar{h}(V+Z)=\left\langle V, j(Z)^{2} V\right\rangle$, where

$$
j(Z)^{2}=\left(\begin{array}{cccc}
-a^{2} & 0 & a b & 0 \\
0 & -a^{2}-b^{2} & 0 & b c \\
a b & 0 & -b^{2}-c^{2} & 0 \\
0 & b c & 0 & -c^{2}
\end{array}\right) .
$$

This induces an invariant first integral on $T N_{P}$ given by $h(p, Y)=\bar{h}(Y)$ as in Proposition 3.3 The gradient of $h$ is given by $\operatorname{grad} h(p, Y)=(0,2([j(Z) V, V]+$ $\left.j(Z)^{2} V\right)$, where $Y=V+Z \in \mathfrak{n}_{P}$. Summarizing, we get the following set of invariant functions in involution:

- $g(p, Y)=\frac{1}{2}\langle Y, Y\rangle$;

- $h(p, Y)=\left\langle Y, j(Z)^{2} Y_{\mathfrak{v}}\right\rangle$, where $Y_{\mathfrak{v}}$ denotes the projection of $Y \in \mathfrak{n}_{P}$ onto $\mathfrak{v}$;

- the three functions for the center $f_{i}(p, Y)=\left\langle Y, Z_{i}\right\rangle$ for $i=1,2,3$.

Invariant functions clearly descend to any space $T\left(\Lambda \backslash N_{P}\right)$ for any cocompact lattice $\Lambda$, since the Lie algebra has rational structure constants. We need two more functions to have the complete integrability (in the Liouville sense) in this case.

Remark 3.5. The coadjoint action of $N$ to $\mathfrak{n}^{*}$ is induced to $\mathfrak{n}$ via the metric, obtaining

$$
p \cdot Y=\operatorname{Ad}^{t}\left(p^{-1}\right)(Y) \text { for all } g \in N, X \in \mathfrak{n},
$$

where $\operatorname{Ad}^{t}(p)$ denotes the transpose of the adjoint map relative to the metric, $\left\langle\operatorname{Ad}^{t}(p)(Y), Y\right\rangle=\langle Y, \operatorname{Ad}(p) X\rangle$ for all $p \in N, X, Y \in \mathfrak{n}$. Also tangent vectors to the orbit are induced by vector fields on $\mathfrak{n}$, that is $X \in \mathfrak{n}$ gives $\tilde{X}(Y)$ :

$$
\tilde{X}(Y)=\left.\frac{d}{d s}\right|_{s=0} \exp (s X) \cdot Y=-\operatorname{ad}^{t}(X)(Y) .
$$

Given a function $F: \mathfrak{n} \rightarrow \mathbb{R}$ one can consider its restriction to a coadjoint orbit. But functions which are not trivial on $\mathfrak{n}$ can become constant on a coadjoint orbit. Take for instance $\mathfrak{n}$ a 2-step nilpotent Lie algebra equipped with a metric and consider the function $F: \mathfrak{n} \rightarrow \mathbb{R}$ given by $F_{0}(Y)=\left\langle Y, Z_{0}\right\rangle$, where $Z_{0} \in \mathfrak{z}$ is a fixed 
vector. It is not hard to see that $\operatorname{grad}_{\mathfrak{n}} F_{0}(Y)=Z_{0}$. But the restriction of $F_{0}$ to the coadjoint orbit gives

$$
d F_{0 Y}(\tilde{X})=\left.\frac{d}{d s}\right|_{s=0}\left\langle\operatorname{Ad}^{t}(\exp -s X)(Y), Z_{0}\right\rangle=-\left\langle Y, \operatorname{ad}(X) Z_{0}\right\rangle=0,
$$

which proves the assertion. Notice that the example holds for any Lie algebra with non-trivial center.

3.1. The situation on $K_{3}$. Here we come back to the 2-step nilpotent Lie group and quotients associated to the complete graph $K_{3}$. The corresponding simply connected Lie group $N_{K_{3}}$ admits a lattice $\Gamma$ and, as already said, no quotient $\Gamma \backslash N_{K_{3}}$ can be endowed with Riemannian metric -induced from a left invariant metric on $N_{K_{3}}$ - such that the corresponding geodesic flow is completely integrable. This question was discussed in [7]. However, the geodesic flow on $N_{K_{3}}$ can be completely integrable.

Take the notation of Example 2.6 Choose the metric on the Lie algebra $\mathfrak{n}_{K_{3}}$ for which the set $V_{1}, V_{2}, V_{3}, Z_{1}, Z_{2}, Z_{3}$ is an orthonormal basis. By Proposition 3.1 one has the set of invariant functions on $T N_{K_{3}}$ given by

$$
\begin{aligned}
& 2 E(p, Y)=\sum_{i=1}^{3}\left\langle Y, V_{i}\right\rangle^{2}+\sum_{i=1}^{3}\left\langle Y, Z_{i}\right\rangle^{2} \\
& f_{Z_{j}}(p, Y)=\left\langle Z_{j}, Y\right\rangle, \quad \text { for } j=1,2,3 .
\end{aligned}
$$

The invariant function

$$
G(p, Y)=\left\langle Y, Z_{1}\right\rangle\left\langle Y, V_{3}\right\rangle+\left\langle Y, Z_{2}\right\rangle\left\langle Y, V_{1}\right\rangle+\left\langle Y, Z_{3}\right\rangle\left\langle Y, V_{2}\right\rangle
$$

is also a first integral of the geodesic flow. The gradient of $G$ is given by

$$
\begin{aligned}
\operatorname{grad} G(p, Y)=\left(0,\left\langle Y, Z_{2}\right\rangle V_{1}\right. & +\left\langle Y, Z_{3}\right\rangle V_{2}+\left\langle Y, Z_{1}\right\rangle V_{3} \\
& \left.+\left\langle Y, V_{3}\right\rangle Z_{1}+\left\langle Y, V_{1}\right\rangle Z_{2}+\left\langle Y, V_{2}\right\rangle Z_{3}\right),
\end{aligned}
$$

so that it is not hard to prove that it satisfies the condition (2.4):

$$
\left\langle Y,\left[Y,\left\langle Y, Z_{2}\right\rangle V_{1}+\left\langle Y, Z_{3}\right\rangle V_{2}+\left\langle Y, Z_{1}\right\rangle V_{3}\right]\right\rangle=0 .
$$

On the other hand it is clear that $G$ is in involution with $f_{Z_{1}}, f_{Z_{2}}, f_{Z_{3}}$.

At this point we have five invariant first integrals which can be induced to the quotient. One needs one more first integral, which can be taken from Killing vector fields. To compute a right-invariant vector field, write the operation on the Lie group, which for coordinates $x_{1}, x_{2}, x_{3}, z_{1}, z_{2}, z_{3}$ is given by

$$
\begin{aligned}
& \left(x_{1}, x_{2}, x_{3}, z_{1}, z_{2}, z_{3}\right)\left(x_{1}^{\prime}, x_{2}^{\prime}, x_{3}^{\prime}, z_{1}^{\prime}, z_{2}^{\prime}, z_{3}^{\prime}\right) \\
& =\left(x_{1}+x_{1}^{\prime}, x_{2}+x_{2}^{\prime}, x_{3}+x_{3}^{\prime}, z_{1}+z_{1}^{\prime}+\frac{1}{2}\left(x_{1} x_{2}^{\prime}-x_{1}^{\prime} x_{2}\right)\right. \text {, } \\
& \left.z_{2}+z_{2}^{\prime}+\frac{1}{2}\left(x_{2} x_{3}^{\prime}-x_{3} x_{2}^{\prime}\right), z_{3}+z_{3}^{\prime}+\frac{1}{2}\left(x_{3} x_{1}^{\prime}-x_{1} x_{3}^{\prime}\right)\right) .
\end{aligned}
$$

So the right-invariant vector field $V_{1}^{*}$ at $p=\left(x_{1}, x_{2}, x_{3}, z_{1}, z_{2}, z_{3}\right)$ is given by $V_{1}^{*}(p)=\partial_{x_{1}}+\frac{1}{2} x_{2} \partial_{z_{1}}-\frac{1}{2} x_{3} \partial_{z_{3}}$, which implies that $f_{V_{1}^{*}}(p, Y)=\left\langle V_{1}+x_{2} Z_{1}-\right.$ $\left.x_{3} Z_{3}, Y\right\rangle$. Its gradient field is

$$
\operatorname{grad} f_{V_{1}^{*}}(p, Y)=\left(\left\langle Y, Z_{1}\right\rangle V_{2}-\left\langle Y, Z_{3}\right\rangle V_{3}, V_{1}+x_{2} Z_{1}-x_{3} Z_{3}\right) .
$$


Straighforward computations show that the functions $f_{Z_{i}}, G, E, f_{V_{1}^{*}}$, for $i=1,2,3$, are linearly independent in a dense subset of $T N_{K_{3}}$.

This proves that the geodesic flow on $T N_{K_{3}}$ is completely integrable. But according to the non-integrability condition on $\mathfrak{n}_{K_{3}}$, the geodesic flow cannot be Liouville integrable on $T\left(\Gamma \backslash N_{K_{3}}\right)$ for any cocompact discrete subgroup $\Gamma<N$.

Remark 3.6. If the smoothly closed geodesics in a nilmanifold $\Gamma \backslash N$ are dense, then the nilmanifold has the density of closed geodesics property. In [9] the authors give conditions on the graph $G$ and on a lattice $\Gamma \subset N$ for which the quotient $\Gamma \backslash N$, a compact nilmanifold, has a dense set of smoothly closed geodesics.

In particular, in the situation of the graph $K_{3}$ the following is proved. Let $\Gamma$ be the lattice in $N_{K_{3}}$ given by $\exp (\Lambda)$, where $\Lambda$ is the vector lattice in $\mathfrak{n}$ given by $\Lambda=\operatorname{span}_{2 \pi \mathbb{Z}}\{\beta\}$, for $\beta$ the orthonormal basis determined by the graph. Then the quotient $\Gamma \backslash N_{K_{3}}$ has the density of closed geodesics property.

\section{GeOdesic FLOW AND GRAPHS}

Here we study the integrability of the geodesic flow on 2-step nilpotent Lie groups arising from graphs. We consider two situations: the family of star graphs on $k+1$ vertices and graphs in $j$ vertices, with $j \leq 4$. We show complete integrability of geodesic flows on compact manifolds induced from star graphs as well as on the corresponding simply connected Lie groups. The corresponding Lie algebras are singular.

For graphs with $j$ vertices, $j \leq 4$, Liouville integrability is proved for the almost non-singular cases.

4.1. Star graphs. Let $S_{k}$ be the star graph on $k+1$ vertices introduced in Example 2.5. Let $N_{S_{k}}$ denote the simply connected 2-step nilpotent Lie group for the Lie algebra associated to it. Consider its presentation by the underlying manifold $\mathbb{R}^{2 k+1}$ as follows. Let $v=\left(x_{0}, x_{1}, \ldots, x_{k}\right)$ and $v^{\prime}=\left(x_{0}^{\prime}, x_{1}^{\prime}, \ldots, x_{k}^{\prime}\right)$ be elements in $\mathbb{R}^{k+1}$; then the group operation on $\mathbb{R}^{2 k+1}$ is given by

$$
\begin{aligned}
\left(v, z_{1}, z_{2}, \ldots, z_{k}\right)\left(v^{\prime}, z_{1}^{\prime}, z_{2}^{\prime}, \ldots, z_{k}^{\prime}\right)= & \left(x_{0}+x_{0}^{\prime}, x_{1}+x_{1}^{\prime}, \ldots, x_{k}+x_{k}^{\prime},\right. \\
& z_{1}+z_{1}^{\prime}+\frac{1}{2}\left(x_{0} x_{1}^{\prime}-x_{0}^{\prime} x_{1}\right), \\
& z_{2}+z_{2}^{\prime}+\frac{1}{2}\left(x_{0} x_{2}^{\prime}-x_{0}^{\prime} x_{2}\right), \\
& \vdots \\
& \left.z_{k}+z_{k}^{\prime}+\frac{1}{2}\left(x_{0} x_{k}^{\prime}-x_{0}^{\prime} x_{k}\right)\right) .
\end{aligned}
$$

Denote by $\partial_{u}$ the partial derivation on $\mathbb{R}^{2 k+1}$ with respect to the variable $u$. A basis of left-invariant vector fields is given by

$$
\begin{aligned}
& V_{0}(p)=\partial_{x_{0}}-\frac{1}{2} x_{1} \partial_{z_{1}}-\frac{1}{2} x_{2} \partial_{z_{2}} \cdots-\frac{1}{2} x_{k} \partial_{z_{k}}, \\
& V_{i}(p)=\partial_{x_{i}}+\frac{1}{2} x_{0} \partial_{z_{i}}, \\
& Z_{i}(p)=\partial_{z_{i}}, \quad \text { for all } i=1, \ldots, k,
\end{aligned}
$$


where $p=\left(x_{0}, x_{1}, \ldots, x_{k}, z_{1}, \ldots, z_{k}\right) \in N$. These vector fields satisfy the nontrivial Lie bracket relations

$$
\left[V_{0}, V_{i}\right]=Z_{i}, \quad \text { for all } i=1,2, \ldots, k .
$$

Consider the metric on $\mathbb{R}^{2 k+1}$ which makes this set into an orthonormal basis. In canonical coordinates such metric is given by

$$
\begin{aligned}
g= & \left(1+\frac{1}{4} \sum_{j=1}^{k} x_{j}^{2}\right) d x_{0}^{2}+\sum_{j=1}^{k}\left(\frac{x_{0} x_{j}}{4} d x_{j}-\frac{x_{j}}{2} d z_{j}\right) d x_{0} \\
& +\sum_{i=1}^{k}\left(1+\frac{x_{0}^{2}}{4}\right) d x_{i}^{2}-\frac{1}{2} \sum_{i=1}^{k} x_{0} d z_{i} d x_{i}+\sum_{i=1}^{k} d z_{i}^{2} .
\end{aligned}
$$

Notice that the exponential map exp $: \mathfrak{n}_{S_{k}} \rightarrow N_{S_{k}}$ is

$$
\exp \left(\sum_{i=0}^{k} x_{i} V_{i}+\sum_{j=1}^{k} z_{j} Z_{j}\right)=\left(x_{0}, x_{1}, \ldots, x_{k}, z_{1}, z_{2}, \ldots, z_{k}\right),
$$

where $V_{i}, Z_{j}$ denote the left-invariant vector fields above.

Any right-invariant vector field on $N_{S_{k}}$ can be regarded as a Killing vector field. In particular, we have the following basis of right-invariant vector fields:

$$
\begin{aligned}
V_{0}^{*}(p) & =\partial_{x_{0}}+\frac{1}{2} x_{1} \partial_{z_{1}}+\frac{1}{2} x_{2} \partial_{z_{2}}+\cdots+\frac{1}{2} x_{k} \partial_{z_{k}}, \\
V_{i}^{*}(p) & =\partial_{x_{i}}-\frac{1}{2} x_{0} \partial_{z_{i}}, \\
Z_{i}^{*}(p) & =\partial_{z_{i}}, \quad \text { for all } i=1, \ldots, k .
\end{aligned}
$$

Notice that $\partial_{z}$ is both left and right-invariant. We induce smooth functions on $T N$ given by

$$
\begin{aligned}
f_{V_{0}^{*}}(p, Y) & =\left\langle V_{0}+\sum_{i=1}^{k} x_{i} Z_{i}, Y\right\rangle, \\
f_{V_{j}^{*}}(p, Y) & =\left\langle V_{j}-\left\langle W, V_{0}\right\rangle Z_{j}, Y\right\rangle, \\
f_{Z_{j}}(p, Y) & =\left\langle Z_{j}, Y\right\rangle,
\end{aligned}
$$

which are first integrals of the geodesic flow, for all $j=1, \ldots, k$ and for $\exp (W)=$ $p \in N_{S_{k}}$. It is not hard to see that for $j=1, \ldots, k$ the corresponding gradient fields are given by

$$
\begin{aligned}
& \operatorname{grad} f_{V_{j}^{*}}(p, Y)=\left(-\left\langle Y, Z_{j}\right\rangle V_{0}, V_{j}-\left\langle W, V_{0}\right\rangle Z_{j}\right) \\
& \operatorname{grad} f_{Z_{j}}(p, Y)=\left(0, Z_{j}\right),
\end{aligned}
$$

which are linearly independent whenever (a) $\left\langle Y, V_{0}\right\rangle \neq 0$ or (b) $\left\langle Y, V_{0}\right\rangle=0$ and $\sum_{j=1}^{k}\left\langle Y, V_{j}\right\rangle\left\langle Y, Z_{j}\right\rangle \neq 0$. This follows from the computations. In fact, for the first component we have $\sum_{i=1}^{k} a_{i}\left\langle Z_{i}, Y\right\rangle=0$, and on the other hand, on the second component:

$$
\sum_{i=1}^{k} a_{i}\left(V_{i}-\left\langle W, V_{0}\right\rangle Z_{i}\right)+\sum_{i=1}^{k} b_{i} Z_{i}+c Y=0 .
$$


Notice that there is only one term involving $V_{0}$ in this equation, and the coefficient is $\left\langle Y, V_{0}\right\rangle$. So if $\left\langle Y, V_{0}\right\rangle \neq 0$ then $c=0$ and so $\sum_{i=1}^{k} a_{i} V_{i}=0$ implies that $a_{i}=0$ for all $i$, and from this $b_{i}=0$ for all $i=1, \ldots, k$. If $\left\langle Y, V_{0}\right\rangle=0$ the condition (b) asserts the linear independence. This proves the first part of the following result.

Lemma 4.1. Let $S_{k}$ denote the star graph on $k+1$ vertices. The smooth functions on $T N_{S_{k}}$ denoted by $f_{V_{j}^{*}}$ (as in equation 4.2) are pairwise in involution for $j=$ $1, \ldots, k$.

Moreover, the geodesic flow on $T N_{S_{k}}$ is completely integrable (in the Liouville sense) since the set of first integrals $\left\{E, f_{Z_{j}}, f_{V_{j}^{*}}\right\}_{j=1}^{k}$ satisfies that any pair of first integrals is in involution and the corresponding gradients are linearly independent on an open dense set.

We only have to prove that $\left\{f_{V_{j}^{*}}, f_{V_{i}^{*}}\right\}=0$ for all $i, j=1, \ldots, k$. In fact, for $i \neq j$ straighforward computations show that

$$
\left\{f_{V_{j}^{*}}, f_{V_{i}^{*}}\right\}(p, Y)=\left\langle Y,\left[V_{i}, V_{j}\right]\right\rangle=0,
$$

which finishes the proof of the lemma.

Note that all first integrals above are polynomial functions of degree one or two. In fact, writing $W=\sum_{i=0}^{k} w_{i} V_{i}+\sum_{i=1}^{k} u_{i} Z_{i}$ and $Y=\sum_{j=0}^{k} y_{j} V_{j}+\sum_{j=1}^{k} z_{j} Z_{j}$, the first integrals follow:

$$
\begin{aligned}
g(p, Y) & =\frac{1}{2}\left(y_{0}^{2}+y_{1}^{2}+\cdots+y_{k}^{2}+z_{1}^{2}+\cdots+z_{k}^{2}\right) \\
f_{V_{1}^{*}}(p, Y) & =y_{1}-w_{0} z_{1} \\
& \vdots \\
f_{V_{k}^{*}}(p, Y) & =y_{k}-w_{0} z_{k} \\
f_{Z_{1}}(p, Y) & =z_{1} \\
& \vdots \\
f_{Z_{k}}(p, Y) & =z_{k} .
\end{aligned}
$$

Remark 4.2. Take coordinates $\left(x_{0}, x_{1}, \ldots, x_{k}, z_{1}, \ldots, z_{k}\right)$ for $p \in N_{S_{k}}$ and coordinates $\left(y_{0}, y_{1}, \ldots, y_{k}, t_{1}, \ldots, t_{k}\right)$ on $\mathfrak{n}_{S_{k}}(=\mathfrak{v} \oplus \mathfrak{z})$ relative to a basis of left-invariant vector fields. Let $F: T N_{S_{k}} \rightarrow \mathbb{R}^{2 k+1}$ be given by $F(p, Y)=\left(E(p, Y), f_{Z_{1}}(p, Y)\right.$, $\left.\ldots, f_{Z_{k}}(p, Y), f_{V_{1}^{*}}(p, Y), \ldots, f_{V_{k}^{*}}(p, Y)\right)$. Let $c \in \mathbb{R}^{2 k+1}$, namely $c=\left(C_{0}, U_{1}, \ldots\right.$, $\left.U_{k}, T_{1}, \ldots, T_{k}\right)$; then the set $F^{-1}(c)$ gives a symplectic leaf on $T N_{S_{k}}$. In fact, whenever $(p, Y) \in F^{-1}(c)$ one has

- $E(p, Y)=\frac{1}{2}\left(y_{0}^{2}+\cdots+y_{k}^{2}+t_{1}^{2}+\cdots+t_{n}^{2}\right)=C_{0}$ and

- $t_{i}=T_{i}$ for $i=1, \ldots, k$ and $y_{j}-x_{0} T_{j}=Y_{j}$, for $j=1, \ldots, k$,

so that there are no restrictions in the coordinates $x_{1}, \ldots, x_{k}, z_{1}, \ldots, z_{k}$ to belong to $F^{-1}(c)$. 
Let $\gamma$ denote a geodesic on $N_{S_{k}}$. Set $\gamma(t)=\exp (X(t)+Z(t))$, where $X(t) \in \mathfrak{v}$ and $Z(t) \in \mathfrak{z}$, with initial condition $X_{0}+Z_{0}$, satisfy the following system of equations $($ see $[10])$ :

$$
\begin{aligned}
x_{0}^{\prime \prime} & =-a_{1} x_{1}^{\prime}-a_{2} x_{2}^{\prime}-\cdots-a_{k} x_{k}^{\prime} \\
x_{1}^{\prime \prime} & =a_{1} x_{0}^{\prime} \\
& \vdots \\
x_{k}^{\prime \prime} & =a_{k} x_{0}^{\prime} \\
z_{1}^{\prime} & =a_{1}+\frac{1}{2}\left(x_{0} x_{1}^{\prime}-x_{0}^{\prime} x_{1}\right) \\
& \vdots \\
z_{k}^{\prime} & =a_{k}+\frac{1}{2}\left(x_{0} x_{k}^{\prime}-x_{0}^{\prime} x_{k}\right),
\end{aligned}
$$

where $Z_{0}=a_{1} Z_{1}+a_{2} Z_{2}+\cdots+a_{k} Z_{k}, X(t)=\sum x_{i}(t) V_{i}$, and $Z(t)=\sum_{j} z_{j}(t) Z_{j}$. The map $j\left(Z_{0}\right)$ shown in Example 2.5 is singular. Its kernel is $\{V \in \mathfrak{v}: V=$ $\sum_{j=0}^{k} v_{j} V_{j}$, where $\left\langle V, V_{0}\right\rangle=0$ and $\left.\left(v_{1}, \ldots, v_{k}\right) \cdot\left(a_{1}, \ldots, a_{k}\right)=0\right\}$, denoting with $\cdot$ the usual inner product for vectors in $\mathbb{R}^{k}$. Thus if $\left\{g^{t}\right\}$ denotes the geodesic flow in $T N$, for every $n \in N$, and $X_{0} \in \mathfrak{v}, Z_{0} \in \mathfrak{z}$, then

$$
g^{t}\left(d L_{n}\left(X_{0}+Z_{0}\right)=d L_{\gamma(t)}\left(e^{t j\left(Z_{0}\right)} X_{0}+Z_{0}\right),\right.
$$

where $\gamma(t)$ denotes the unique geodesic with $\gamma^{\prime}(0)=d L_{n}\left(X_{0}+Z_{0}\right)$.

A Riemannian compact manifold arises as a quotient $\Lambda \backslash N_{S_{k}}$, where $\Lambda$ is a discrete cocompact subgroup of $N_{S_{k}}$. In fact, $\Lambda \backslash N_{S_{k}}$ becomes a Riemannian manifold with the metric that makes the projection $\pi: N_{S_{k}} \rightarrow \Lambda \backslash N_{S_{k}}$ a Riemannian submersion.

Each $(2 k+1)$-tuple $(r, m)=\left(r, r_{1}, \ldots, r_{k}, m_{1}, \ldots, m_{k}\right) \in(\mathbb{Z})^{2 k+1}$ defines a lattice in $N_{S_{k}}$ by

$$
\Lambda_{(r, m)}=r m_{0} \mathbb{Z} \times 2 r_{1} \mathbb{Z} \times \cdots \times 2 r_{k} \mathbb{Z} \times m_{1} \mathbb{Z} \times m_{2} \mathbb{Z} \times \cdots \times m_{k} \mathbb{Z},
$$

for $m_{0}=m_{1} m_{2} \ldots m_{k}$.

Note that there are non-isomorphic lattices in this family so that we get many non-diffeomorphic compact manifolds.

Since the quotient projection $\pi: N_{S_{k}} \rightarrow \Lambda_{(r, m)} \backslash N_{S_{k}}$ is a Riemannian submersion and furthermore a local isometry, we can identify the tangent bundle $T\left(\Lambda_{(r, m)} \backslash N_{S_{k}}\right)$ with $\left(\Lambda_{(r, m)} \backslash N_{S_{k}}\right) \times \mathfrak{n}_{S_{k}}$. The projection $\pi$ maps geodesics into geodesics and the energy function $\tilde{E}$ on $T\left(\Lambda_{(r, m)} \backslash N_{S_{k}}\right)$ is related to the energy function $E$ on $T N_{S_{k}}$ by

$$
\tilde{E}\left(\Lambda_{(r, m)} p, Y\right)=E(p, Y)=\frac{1}{2}\langle Y, Y\rangle
$$

and clearly it is well defined.

All invariant first integrals on $T N_{S_{k}}$ descend to the quotients, since they do not depend on the coordinates of $p \in N_{S_{k}}$. One defines

$$
\tilde{f}_{Z_{j}}\left(\Lambda_{(r, m)} p, Y\right)=f_{Z_{j}}(p, Y), \quad \text { for all } j=1, \ldots, k,
$$


which are first integrals of the geodesic flow of $T\left(\Lambda_{(r, m)} \backslash N_{S_{k}}\right)$. Moreover, such first integrals are in involution, since for all $f, g \in C^{\infty}\left(T\left(\Lambda_{(r, m)} \backslash N_{S_{k}}\right)\right)$ we have

$$
\{f \circ \pi, g \circ \pi\}=\{f, g\} \circ \pi .
$$

Note that the integrals $f_{V_{j}^{*}}, j=1, \ldots, k$, do not descend directly to the quotient. However, one can construct first integrals on the quotient with the following argument. Let $(p, Y) \in T N_{S_{k}}$ and $q \in \Lambda_{(r, m)}$. Take $W, W^{\prime} \in \mathfrak{n}_{S_{k}}$ such that $\exp W=p$, $\exp W^{\prime}=q$. Observe that $\left(W+W^{\prime}\right)_{\mathfrak{v}}=W_{\mathfrak{v}}+W_{\mathfrak{v}}^{\prime}$, where $U_{\mathfrak{v}}$ denotes the orthogonal projection of $U \in \mathfrak{n}_{S_{k}}$ over $\mathfrak{v}=\mathfrak{z}^{\perp}$. So we get

$$
\begin{aligned}
f_{V_{j}^{*}}(q p, Y) & =\left\langle Y, V_{j}\right\rangle-\left\langle Z_{j}, Y\right\rangle\left\langle\left(W+W^{\prime}\right), V_{0}\right\rangle \\
& =f_{V_{j}^{*}}(p, Y)-f_{Z_{j}}(p, Y)\left\langle W^{\prime}, V_{0}\right\rangle .
\end{aligned}
$$

Since $\left\langle W^{\prime}, V_{0}\right\rangle \in \mathbb{Z}$, we have

$$
f_{V_{j}^{*}}(q p, Y)=f_{V_{j}^{*}}(p, Y) \bmod f_{Z_{j}}(p, Y) \mathbb{Z}
$$

for every $j=1, \ldots, k$, and since $f_{Z_{j}}$ is a first integral of the geodesic flow, we have that the function

$$
\hat{f}_{V_{j}^{*}}(p, Y)=\sin \left(2 \pi \frac{f_{V_{j}^{*}}(p, Y)}{f_{Z_{j}}(p, Y)}\right)
$$

descends to $\Lambda_{(r, m)} \backslash N_{S_{k}}$ and is constant along the integral curves of the geodesic vector field in $T\left(\Lambda_{(r, m)} \backslash N_{S_{k}}\right)$. In order to get a smooth first integral let

$$
\bar{F}_{j}(p, Y)=e^{-1 / f_{Z_{j}}(p, Y)^{2}} \hat{f}_{j}(p, Y)
$$

and let us define

$$
\tilde{F}_{j}\left(\Lambda_{r} p, Y\right)=\bar{F}_{j}(p, Y)
$$

So the functions $\tilde{F}_{k}$ are smooth (non-analytic) first integrals for the geodesic flow on $T\left(\Lambda_{(r, m)} \backslash N_{S_{k}}\right)$. It follows, from a direct calculation making use of properties of the Poisson bracket, that the families $f_{Z_{i}}, \tilde{F}_{j}, i=1, \ldots, k$, are in involution. In fact, for a pair of differentiable functions on $M$ and for $h: \mathbb{R} \rightarrow \mathbb{R}$, one has $\{f, h \circ g\}=$ $h^{\prime}\{f, g\}$. To prove the linear independence, notice that the new gradients can be written in terms of the gradients on $N_{S_{k}}$, which are multiplied by differentiable real functions. The independence follows by asking that the corresponding determinant be non-trivial on the right open set. So the geodesic flow in $T\left(\Lambda_{(r, m)} \backslash N_{S_{k}}\right)$ is completely integrable in the sense of Liouville.

Theorem 4.3. Let $N_{S_{k}}$ be the 2-step nilpotent Lie group attached to the star graph in $k+1$ vertices $S_{k}$, endowed with the standard metric, and let $\Lambda_{(r, m)}$ denote the lattice in 4.3. If $\Lambda_{(r, m)} \backslash N_{S_{k}}$ is the corresponding compact manifold with the induced metric, then the geodesic flow in $T\left(\Lambda_{(r, m)} \backslash N_{S_{k}}\right)$ is completely integrable with smooth first integrals $\left\{E, f_{Z_{i}}, \tilde{F}_{i}\right\}$, for $i=1, \ldots, k$.

See explanations on the proof and the topology of these compact manifolds in [6], where the author worked on the cotangent setting. Another difference is the presentation of the Lie group. See the isomorphism in equation 4.4 below. 
Let $\tilde{H} \subset N_{S_{k}}$ be the normal subgroup of dimension $2 k$ defined as $\tilde{H}=\left\{g \in N_{S_{k}}\right.$ : $g=(v, z)$ and $\left.v=\left(0, x_{1}, \ldots, x_{k}\right)\right\}$. Note that $\tilde{H}$ is abelian and $\tilde{\Lambda}_{(r, m)}=\tilde{H} \cap \Lambda_{(r, m)}$ is a lattice in $\tilde{H}$. So $\tilde{\Lambda}_{(r, m)} \backslash \tilde{H} \simeq T^{2 k}$.

Note that $N_{S_{1}}$ is (isomorphic to) the Heisenberg Lie group $H_{3}$. A known presentation of $\mathrm{H}_{3}$ is given in terms of matrices as the set

$$
\left\{\left(\begin{array}{lll}
1 & x & z \\
0 & 1 & y \\
0 & 0 & 1
\end{array}\right) \text { for } x, y, z \in \mathbb{R}\right\}
$$

together with the usual product of matrices. The subgroup $\Gamma_{r}$ consisting of matrices of the form

$$
\Gamma_{r}=\left\{\left(\begin{array}{ccc}
1 & r n & q \\
0 & 1 & m \\
0 & 0 & 1
\end{array}\right) \text { for } m, n, q \in \mathbb{Z}\right\}
$$

for a fixed $r \in \mathbb{N}$ gives rise to a cocompact lattice in $H_{3}$. The lattice $\Gamma_{r}$ induces an action on $H_{3}$ so that the class of $(x, y, z) \in H_{3}$ is $\overline{(x, y, z)}=\{(x+r n, y+m, z+$ $r n+s): n \in \mathbb{Z}, m \in \mathbb{Z}, s \in \mathbb{Z}\}$.

Denoting by $\bar{\Gamma}_{r}$ the subgroup isomorphic to $\mathbb{Z}$ and by $\tilde{\Gamma}$ the subgroup isomorphic to $\mathbb{Z}^{2}$ given respectively by

$$
\bar{\Gamma}_{r}=\{(r n, 0,0): n \in \mathbb{Z}\} \simeq r \mathbb{Z}, \quad \tilde{\Gamma}=\{(0, m, q): m, q \in \mathbb{Z}\} \simeq \mathbb{Z} \times \mathbb{Z},
$$

we get the semidirect product group $\bar{\Gamma}_{r} \ltimes \tilde{\Gamma}$, where the action is given by $r n$. $(m, q)=(m, q+r n m)$, via identifications. So the map $\Psi: \bar{\Gamma}_{r} \ltimes \tilde{\Gamma} \rightarrow \Gamma_{r}$ given as $(r n,(m, q)) \rightarrow(r n, m, q)$ is a group isomorphism, and the action of $\Gamma_{r}$ on $H_{3}$ by translations on the left is equivalent to an action of $\bar{\Gamma}_{r} \ltimes \tilde{\Gamma}$ on $H_{3}$.

Now the action of $\tilde{\Gamma}$ on $H_{3}$ gives

$$
(0, m, q) \cdot(x, y, z)=(x, y+m, z+q), \quad \text { so that } \tilde{\Gamma} \backslash H_{3} \simeq \mathbb{R} \times T^{2} .
$$

One also has

$$
(r n, 0,0) \cdot(0, m, q) \cdot(x, y, z)=(0, m, q) \cdot(r n, 0,0) \cdot(x, y, z)
$$

Finally, the action of $\bar{\Gamma}_{r}$ on $\mathbb{R} \times T^{2}$ induces the action of $S^{1}$ on $\mathbb{R} \times T^{2}$ and $\Gamma_{r} \backslash H_{3}$ is a $S^{1}$-fiber bundle over $T^{2}$ :

$$
S^{1} \rightarrow \Gamma_{r} \backslash H_{3} \rightarrow T^{2}
$$

A similar procedure generalizes to $H_{2 n+1}$ showing that Heisenberg nilmanifolds $\Gamma \backslash H_{2 n+1}$, as topological spaces, are $T^{n}$-fiber bundles over $T^{n+1}$ (see [1]).

Analogously, one shows the fibration we get from the nilmanifolds arising from every star graph. In fact, one defines similar subgroups $\Gamma_{r}, \bar{\Gamma}_{r}$, and $\tilde{\Gamma}$ in $N_{S_{k}}$. This is explained above. 
A presentation of $N_{S_{k}}$ is given by the $(k+2) \times(k+2)$-matrices of the form

$$
\left\{\left(\begin{array}{cccccc}
1 & x_{0} & z_{1} & z_{2} & \ldots & z_{k} \\
0 & 1 & x_{1} & x_{2} & \ldots & x_{k} \\
0 & 0 & 1 & 0 & \ldots & 0 \\
0 & 0 & 0 & 1 & \ldots & 0 \\
0 & & & 0 & \ddots & \vdots \\
0 & & & & & 1
\end{array}\right)\right\}
$$

where we think in the usual matrix multiplication. The map $\Psi$ sending

$$
\left(\begin{array}{cccccc}
1 & x_{0} & z_{1} & z_{2} & \ldots & z_{k} \\
0 & 1 & x_{1} & x_{2} & \ldots & x_{k} \\
0 & 0 & 1 & 0 & \ldots & 0 \\
0 & 0 & 0 & 1 & \ldots & 0 \\
0 & & & 0 & \ddots & \vdots \\
0 & & & & & 1
\end{array}\right) \mapsto\left(x_{0}, x_{1}, x_{2}, \ldots, x_{k}, z_{1}-\frac{1}{2} x_{0} x_{1}, \ldots, z_{k}-\frac{1}{2} x_{0} x_{k}\right)
$$

gives an isomorphism between the above group and the one defined with the multiplication operation of equation (4.1).

For a fixed $r$, take the lattice $\Gamma_{r} \subset N_{S_{k}}$ given by matrices of the form

$$
\Gamma_{r}=\left\{\left(\begin{array}{cccccc}
1 & r n & q_{1} & q_{2} & \cdots & q_{k} \\
0 & 1 & m_{1} & m_{2} & \cdots & m_{k} \\
0 & 0 & 1 & 0 & \cdots & 0 \\
0 & 0 & 0 & 1 & \cdots & 0 \\
0 & & & 0 & \ddots & \vdots \\
0 & & & & & 1
\end{array}\right) \text {, for } n, m_{i}, q_{j} \in \mathbb{Z}, \forall i, j\right\} .
$$

One starts with the abelian subgroup $\tilde{\Gamma}=\left\{\left(0, m_{1}, m_{2}, \ldots, m_{k}, q_{1}, q_{2}, \ldots, q_{k}\right)\right.$ : $\left.m_{i}, q_{j} \in \mathbb{Z}\right\}$, which acts on the left on $N_{S_{k}}$ by

$$
\begin{aligned}
\left(0, m_{1}, m_{2}, \ldots, m_{k}, q_{1}, q_{2}, \ldots, q_{k}\right) \cdot\left(x_{0}, x_{1}, \ldots, x_{k}, z_{1}, \ldots, z_{k}\right) \\
=\left(x_{0}, x_{1}+m_{1}, \ldots, x_{k}+m_{k}, z_{1}+q_{1}, \ldots, z_{k}+q_{k}\right) .
\end{aligned}
$$

This shows that $\tilde{\Gamma} \backslash N_{S_{k}} \simeq \mathbb{R} \times T^{2 n}$. Let $X_{0} \in N_{S_{k}}$ denote the element $X_{0}=$ $\left(x_{0}, x_{1}+m_{1}, \ldots, x_{k}+m_{k}, z_{1}+q_{1}, \ldots, z_{k}+q_{k}\right)$ and take the action of $\bar{\Gamma}_{r}$ on $\tilde{\Gamma} \backslash N_{S_{k}}$ where $\bar{\Gamma}_{r}=\{(r n, 0, \ldots, 0): n \in \mathbb{Z}\}<N_{S_{k}}$ :

$$
\begin{aligned}
(r n, 0, \ldots, 0) \cdot X_{0}= & \left(x_{0}+r n, x_{1}+m_{1}, \ldots, x_{k}+m_{k},\right. \\
& \left.z_{1}+q_{1}+r n x_{1}+r n m_{1}, \ldots, z_{k}+q_{k}+r n x_{k}+r n m_{k}\right) .
\end{aligned}
$$

As above for $H_{3}$ one has $\Gamma_{r} \simeq \bar{\Gamma}_{r} \times \tilde{\Gamma}$ and the action of $\Gamma_{r}$ on $N_{S_{k}}$ translates into an action of $\bar{\Gamma}_{r} \ltimes \tilde{\Gamma}$ on $N_{S-k}$, showing that the compact manifold $\Gamma_{r} \backslash N_{S_{k}}$ is a $S^{1}$-fiber bundle over $T^{2 k}$ :

$$
S^{1} \rightarrow \Gamma_{r} \backslash N_{S_{k}} \rightarrow T^{2 n}
$$


The action of $S^{1}$ on each torus $T^{2}$ is induced by the matrix action on each subspace $\left(z_{i}, x_{i}\right)$ for $i=1, \ldots, k$ :

$$
\left(\begin{array}{ll}
1 & r \\
0 & 1
\end{array}\right)
$$

as already explained on the Heisenberg Lie group $H_{3}$.

Remark 4.4. Let $\varphi_{t}$ denote the geodesic flow. It was proved in [6] that $\varphi_{t}$ is non-degenerate in the sense of KAM theory. Moreover, the topological entropy of $\varphi_{t}$ vanishes. Indeed, $\pi_{1}\left(\Gamma_{1} \backslash N_{S_{k}}\right)$ has no abelian subgroup of finite index. For more information on the topology see [6].

4.2. Non-commutative integrability and graphs. Here we prove the integrability of geodesic flows on manifolds associated to graphs in a low number $k$ of vertices, where $k \leq 4$.

Let $H: T N \rightarrow \mathbb{R}$ denote a smooth function. One says that $H$ is integrable in the non-commutative sense of Nekhorosev, or simply integrable, if one has the following conditions. Assume that $F=\left(H=f_{1}, \ldots, f_{n-k}, g_{1}, \ldots, g_{2 k}\right)$ is a smooth map on $T N$, where $\operatorname{dim} N=n$ and $k \geq 0$, that satisfies the three conditions:

(i) $\operatorname{rank} d F=n+k$ on an open, dense subset of $T N$;

(ii) for all $a, b=1, \ldots, n-k$ and all $c=1, \ldots, 2 k:\left\{f_{a}, f_{b}\right\}=\left\{f_{a}, g_{c}\right\}=0$;

(iii) for each regular value $c \in \mathbb{R}^{n+k}$, each connected component of $F^{-1}(c)$ is compact.

In this situation, the Theorem of Nekhorosev describes the level sets of $F$ and the flow for the Hamiltonian $X_{H}$ in terms of a flow on the torus. In [5] Butler proves integrability in the non-commutative sense of the geodesic flow for $D \backslash N$, where $D$ is a cocompact lattice on $N$ and $N$ is a 2-step nilpotent Lie group whose Lie algebra is almost non-singular. In fact, one can find $3 s+t$ first integrals, where $\operatorname{dim} \mathfrak{v}=2 s$ and $\operatorname{dim} \mathfrak{z}=t$. These functions can be obtained in both ways studied in this section: invariant functions from Proposition 3.3 and the $n$ functions arising from a basis of right-invariant vector fields.

Since Bolsinov and Jovanović proved that integrability in the non-commutative sense implies Liouville integrability (see [1]), the previous results of Butler give the Liouville integrability for an important family of 2-step nilpotent Lie groups and their compact quotients.

Let us explain the construction. Let $N$ denote a Lie group equipped with a left-invariant metric and with Lie algebra $\mathfrak{n}=\mathfrak{v} \oplus \mathfrak{z}$, with $\operatorname{dim} \mathfrak{v}=2 n$, $\operatorname{dim} \mathfrak{z}=m$. Making use of Killing vectors we construct $2 n+m$ first integrals of the geodesic flow. With Proposition 3.3 we construct $n$ invariant first integrals. Assume that this is a Lie algebra. In this Lie algebra we have $n+m$ first integrals which are in involution. So that we get $2 n+m+n+n+m=2 m+4 n=\operatorname{dim} T N$. This gives the complete integrability for almost non-singular Lie algebras. As a corollary we obtain the next result.

Corollary 4.5. Let $G$ denote a connected graph on $k$ vertices with $k \leq 4$. Then, except for the complete graph $K_{3}$, any 2-step nilpotent Lie group $N_{G}$ so as the corresponding compact quotient admits a completely integrable geodesic flow. 
Take the graphs in Example 2.13 For two vertices, we have the Heisenberg Lie algebra of dimension three whose geodesic flow is completely integrable with any left-invariant metric. For three vertices, the connected graphs are $S_{3}$ and $K_{3}$, which were explained above. We need to concentrate in algebras coming from graphs with four vertices.

Example 4.6. Let $P$ be the path in four vertices. Let $N_{P}$ denote the 2 -step nilpotent Lie group associated to $P$. Then $N_{P}$ has dimension seven. Take $p=$ $\exp (W)$ and the functions on $T N_{P}$ given by

$$
\begin{aligned}
& f_{V_{1}^{*}}(p, Y)=\left\langle V_{1}+\left\langle W, V_{2}\right\rangle Z_{1}, Y\right\rangle, \\
& f_{V_{2}^{*}}(p, Y)=\left\langle V_{2}-\left\langle W, V_{1}\right\rangle Z_{1}+\left\langle W, V_{3}\right\rangle Z_{3}, Y\right\rangle, \\
& f_{V_{3}^{*}}(p, Y)=\left\langle V_{3}-\left\langle W, V_{2}\right\rangle Z_{2}+\left\langle W, V_{4}\right\rangle Z_{3}, Y\right\rangle, \\
& f_{V_{4}^{*}}(p, Y)=\left\langle V_{4}-\left\langle W, V_{3}\right\rangle Z_{3}, Y\right\rangle .
\end{aligned}
$$

In this situation we do not need the functions of Proposition 3.3 In fact, Killing vectors obtained as right-invariant vector fields give rise to a subalgebra of first integrals of dimension 7. Among these first integrals, we have three from the center which are in involution and also two more functions, $f_{V_{1}^{*}}$ and $f_{V_{4}^{*}}$, which are in involution. In this situation, since $\mathfrak{n}_{P}$ is almost non-singular, we could apply the result in [1] to get the complete integrability. But we are also able to give explicitly a family of first integrals in involution. In fact, we already have five first integrals in involution as in Example 3.4 . Choose the first integrals above, $f_{V_{1}^{*}}$ and $f_{V_{4}^{*}}$. It is not hard to prove that the corresponding gradient fields are given by

$$
\begin{aligned}
& \operatorname{grad} f_{V_{1}^{*}}(p, Y)=\left(\left\langle Y, Z_{1}\right\rangle V_{2}, V_{1}+\left\langle W, V_{2}, V_{2}\right\rangle Z_{1}\right), \\
& \operatorname{grad} f_{V_{4}^{*}}(p, Y)=\left(-\left\langle Y, Z_{3}\right\rangle V_{3}, V_{4}-\left\langle W, V_{3}\right\rangle Z_{3}\right) .
\end{aligned}
$$

Making use of this information and by doing similar computations as already shown for Lie groups associated to star graphs, one can prove the following:

The set $E, h, f_{Z_{1}}, f_{Z_{2}}, f_{Z_{3}}, f_{V_{1}^{*}}, f_{V_{4}^{*}}$ is a set of first integrals in involution.

The map $h$ above was defined in Example 3.4 .

\section{ACKNowledgment}

The author thanks the generous comments and suggestions of the referee to improve the first version of the work.

\section{REFERENCES}

[1] A. V. Bolsinov and B. Jovanović, Noncommutative integrability, moment map and geodesic flows, Ann. Global Anal. Geom. 23 (2003), no. 4, 305-322. MR 1972543.

[2] A. V. Bolsinov and B. Jovanović, Complete involutive algebras of functions on cotangent bundles of homogeneous spaces, Math. Z. 246 (2004), no. 1-2, 213-236. MR 2031454

[3] A. V. Bolsinov and I. A. Taimanov, An example of an integrable geodesic flow with positive topological entropy, Russian Math. Surveys 54 (1999), no. 4, 833-834, translation from Uspekhi Mat. Nauk 54 (1999), no. 4(328), 157-158. MR 1741286.

[4] A. V. Bolsinov and I. A. Taimanov, Integrable geodesic flows with positive topological entropy, Invent. Math. 140 (2000), no. 3, 639-650. MR 1760753 
[5] L. Butler, Integrable geodesic flows with wild first integrals: the case of two-step nilmanifolds, Ergodic Theory Dynam. Systems 23 (2003), no. 3, 771-797. MR 1992663

[6] L. T. Butler, New examples of integrable geodesic flows, Asian J. Math. 4 (2000), no. 3, 515-526. MR 1796692

[7] L. T. Butler, Zero entropy, non-integrable geodesic flows and a non-commutative rotation vector, Trans. Amer. Math. Soc. 355 (2003), no. 9, 3641-3650. MR 1990166.

[8] S. G. Dani and M. G. Mainkar, Anosov automorphisms on compact nilmanifolds associated with graphs, Trans. Amer. Math. Soc. 357 (2005), no. 6, 2235-2251. MR 2140439

[9] R. C. DeCoste, L. DeMeyer and M. G. Mainkar, Graphs and metric 2-step nilpotent Lie algebras, Adv. Geom. 18 (2018), no. 3, 265-284. MR 3830180

[10] P. Eberlein, Geometry of 2-step nilpotent groups with a left invariant metric, Ann. Sci. École Norm. Sup. (4) 27 (1994), no. 5, 611-660. MR 1296558

[11] P. Eberlein, Left invariant geometry of Lie groups, Cubo 6 (2004), no. 1, 427-510. MR 2077156

[12] C. S. Gordon, Y. Mao and D. Schueth, Symplectic rigidity of geodesic flows on two-step nilmanifolds, Ann. Sci. École Norm. Sup. (4) 30 (1997), no. 4, 417-427. MR 1456241

[13] R. Gornet and M. B. Mast, The length spectrum of Riemannian two-step nilmanifolds, Ann. Sci. École Norm. Sup. (4) 33 (2000), no. 2, 181-209. MR 1755115

[14] A. Kocsard, G. P. Ovando and S. Reggiani, On first integrals of the geodesic flow on Heisenberg nilmanifolds, Differential Geom. Appl. 49 (2016), 496-509. MR 3573846

[15] B. Kostant, The solution to a generalized Toda lattice and representation theory, Adv. in Math. 34 (1979), no. 3, 195-338. MR 0550790.

[16] M. G. Mainkar, Graphs and two-step nilpotent Lie algebras, Groups Geom. Dyn. 9 (2015), no. 1, 55-65. MR 3343346

[17] A. I. Malcev, On a class of homogeneous spaces, Amer. Math. Soc. Transl., no. 39, 1951. MR 39734 .

[18] G. P. Paternain and R. J. Spatzier, New examples of manifolds with completely integrable geodesic flows, Adv. Math. 108 (1994), no. 2, 346-366. MR 1296518

[19] D. Schueth, Integrability of geodesic flows and isospectrality of Riemannian manifolds, Math. Z. 260 (2008), no. 3, 595-613. MR 2434471

[20] W. W. Symes, Systems of Toda type, inverse spectral problems, and representation theory, Invent. Math. 59 (1980), no. 1, 13-51. MR 0575079

[21] I. A. Taŭmanov, Topological obstructions to integrability of geodesic flows on non-simplyconnected manifolds, Math. USSR-Izv. 30 (1988), no. 2, 403-409, translation from Izv. Akad. Nauk SSSR Ser. Mat. 51 (1987), no. 2, 429-435, 448. MR 0897007

[22] I. A. Taŭmanov, Topology of Riemannian manifolds with integrable geodesic flows, Proc. Steklov Inst. Math. 4(205) (1995), 139-150, translation from Tr. Mat. Inst. Steklova 205 (1994), 150-163. MR 1428676

[23] A. Thimm, Integrable geodesic flows on homogeneous spaces, Ergodic Theory Dynam. Systems 1 (1981), no. 4, 495-517. MR 0662740.

Gabriela P. Ovando

CONICET-UNR, Departamento de Matemática, ECEN - FCEIA, Pellegrini 250, 2000 Rosario, Santa Fe, Argentina

gabriela@fceia.unr.edu.ar

Received: November 30, 2018

Accepted: August 6, 2019 